\title{
Estrés laboral y desempeño en docentes de Instituciones Educativas Públicas
}

DOI: $10.46932 / \mathrm{sfjdv2n1-044}$

Received in: November 1st, 2020

Accepted in: December 30th, 2020

\author{
Helen Catalina Rabanal-León \\ Lic. en Educación- Especialidad de matemáticas \\ Lic. En Administración \\ Mg. En Psicología Educativa \\ Universidad César Vallejo, Trujillo, Perú. \\ Villa Agraria, Pasaje Las Flores. Mz. "C". Lote "3" Chepén \\ E-mail: hrabanal@ucv.edu.pe \\ Irma Luz Yupari-Azabache \\ Lic. en Estadística \\ Dr. en Administración de la Educación \\ Instituto de Investigación - Universidad César Vallejo, Trujillo, Perú. \\ Av. Víctor Larco 1770 \\ E-mail: IYUPARI@ucv.edu.pe
}

\author{
Pablo Valentino Aguilar-Sánchez \\ Ing. Estadístico \\ MBA en Administración de Negocios. \\ Universidad César Vallejo, Trujillo, Perú. \\ La Perla 476. Urb. Santa Inés. \\ E-mail: pvaguilarc@ucvvirtual.edu.pe
}

\author{
Nelly Roxana Carranza Yuncor \\ Lic. en Educación Primaria \\ Mg. en Psicología Educativa. \\ Universidad César Vallejo, Trujillo, Perú. \\ E-mail: ncarranzay25@ucvvirtual.edu.pe
}

\section{RESUMEN}

La presente investigación permitió determinar si los docentes de tres instituciones educativas de Chepén presentan síntomas de estrés que perjudiquen el desempeño de su trabajo, sabiendo que es lo principal dentro de una institución para alcanzar sus metas y objetivos, y que mejorar este aspecto permitirá que las instituciones puedan llegar al éxito. El objetivo principal fue determinar si existe relación entre el estrés laboral y el desempeño de las Instituciones Educativas de Chepén - 2018. La metodologíá utilizada tuvo un diseño no experimental, de corte transversal y correlacional, cuya muestra estuvo conformada por 125 docentes de tres Instituciones Educativas de Chepén. Los instrumentos utilizados fueron 2 cuestionarios uno para estrés y otro para desempeño, ambos debidamente válidos y confiables. Los resultados obtenidos evidenciaron que existe una relación significativa e inversa entre las variables $(R:-0,212, p<0.05)$ y dentro de las dimensiones se encontró asociación solo entre la dimensión apoyo en el trabajo y el desempeño 
laboral de los docentes de las Instituciones ( $R$ : $-0,21, \mathrm{p}<0.05)$ concluyendo que, si aumenta el estrés laboral en el docente, su desempeño laboral disminuye.

Palabras clave: Estrés laboral, desempeño, docente, instituciones de enseñanza.

\section{INTRODUCCIÓN}

En el Perú, la Ley de la Reforma Magisterial (2013) define al docente como un profesional competente, certificado, agente fundamental del proceso de enseñanza aprendizaje, con capacidad para brindar un servicio de calidad, pertinente y equitativo con la finalidad de formarlo integralmente y contribuyendo, de esta manera, con la comunidad educativa.

Ser docente constituye una de las profesiones más significativas en la evolución del individuo ya que se configura como guía y responsable de transmitir conocimientos, desarrollar competencias y habilidades en los estudiantes dentro de un ambiente propicio; por ello el proceso educativo debe integrar factores que van más allá del desarrollo de saberes y que involucren valores, emociones y expectativas que logren integrar este proceso y hacer que los resultados sean óptimos.

Dentro del marco de la reforma magisterial se establecen también, deberes, que si bien es cierto, buscan mejorar la calidad educativa y labor pedagógica, debido a que los factores que influyen en este proceso ha sido inconstante en la última década y, sumado a ello, una capacitación deficiente y centralizada para este perfeccionamiento y, por otro lado, la poca motivación y/o compromiso de parte de algunos docentes para mejorar su labor pedagógica, ha generado un efecto negativo en su salud psicológica, causando un estrés que repercute en su desempeño laboral dentro y fuera del aula, por ello, muchas veces, las competencias esperadas no llegan a satisfacer a los estudiantes y por ende, a la comunidad educativa y al Estado. (Ley de la Reforma Magisterial, 2013)

Diversos estudios se preocuparon en indagar sobre los factores psicosociales que influyen en el entorno laboral, esto surge por la relación que existe entre dichos factores y sus efectos, por ello es necesario un modelo teórico que delimite la relación entre ambas. (Luceño et al., 2005).

Para autores como Noblet et al., (2006), el estrés laboral se manifiesta cuando se supera la capacidad física o mental del individuo por exceso o exigencia de la carga laboral. Mientras que para Stavroula et al. (2004), el estrés laboral es la reacción del individuo que asume por la presión y exigencia laboral para las cuales no está preparado tanto en conocimientos o capacidades y le impide cumplir una determinada situación, esta situación puede agravarse si no recibe apoyo de sus compañeros o supervisor. No debemos confundir la presión con el estrés, pues la primera, en un nivel aceptable, puede mantener en alerta, motivado y en condiciones favorables para seguir aprendiendo, pero si esta presión se vuelve excesiva o difícil de controlar aparecerá el estrés. 
Según Ontiveros et al., (2019), nos indica que "el término estrés ha sido controversial desde que lo introdujo el fisiólogo canadiense Hans Selye, quien lo define como una respuesta del individuo producida por un estímulo o agente que le produce una situación de estrés.

También, Rodríguez et al., (2017) investigaron sobre el fenómeno del estrés laboral en su forma más aguda (Burnout), en docentes de nivel secundaria, donde concluyen que la sobrecarga laboral repercute en la salud del docente y su entorno, las causas y consecuencias son variadas, diversas y pueden ser exclusivas, es decir, lo que perjudica a un individuo no necesariamente afecta a otro de la misma manera.

Asimismo, Muñoz et al., (2020) manifiestan qué, si el estrés no es atendido a tiempo, éste se puede volver crónico, una característica del burnout debido a que los docentes están sometidos a una sobrecarga laboral y realizan actividades en simultáneo por ello disponen de poco tiempo para sus asuntos personales.

Ahora bien, Acosta et al., (2019) en su investigación, observaron que en los docentes universitarios donde aplicaron su estudio, no presentan ningún indicador que pueda predecir el nivel de estrés, pero manifiestan que desde hace mucho tiempo la docencia es una ocupación con mayor nivel de estrés que otras profesiones. Asimismo, Zuniga et al., (2018) analizaron el nivel de estrés a docentes de un colegio público regional de Chile, encontrando que dichos docentes tenían un nivel medio de estrés.

En la investigación de Lemos et al., (2019) hallaron relación en varios factores como: nivel de estrés percibido, excesivas tareas, trabajo en casa y la intervención de la familia-trabajo y viceversa; así como la asociación negativa del estrés y el control que perciben el individuo sobre el tiempo de docencia y el deseo de tiempo libre en el fin de semana.

Para Gallardo-López et al., (2019), El síndrome de Burnout se determina por la pérdida del disfrute del trabajo, desgaste emocional y se produce cuando las exigencias de sus competencias laborales sobrepasan la capacidad del individuo para desarrollarlas. En la investigación realizada a 324 expertos de 54 países del mundo sobre el estrés en el trabajo, más del 90\% estaba de acuerdo que es una preocupación para su país, 70\% coincidió en que el estrés se relaciona con el trabajo, en otros, en el sector educativo. (Organización Internacional del Trabajo, 2016)

Cuando un individuo se encuentra bajo estrés le dificulta mantener una armonía entre su vida laboral y personal, por ello es importante que las organizaciones e instituciones presten atención a este problema y busquen estrategias adecuadas que sumadas a una buena gestión sean la mejor forma de prevenir el estrés.

Para Leal (2006), los tipos de estrés son tres; el estrés agudo, se da cuando las presiones son causadas por acciones recientes o anticipadas el futuro, el estrés episódico se presenta cuando la persona vivencia con frecuencia el estrés agudo, generalmente son individuos con exceso de trabajo, actividades, 
compromisos, y; por último el estrés crónico, se produce cuando se está mucho tiempo en una situación estresante, pobreza, violencia familiar o realizar un trabajo que le parece desagradable, este tipo de estrés aparece cuando el individuo considera que no puede salir de dicha situación o que es de nunca acabar.

Diversas investigaciones de estrés laboral (Chiang et al., 2013; Fernández-Arata et al., 2017; Carrillo-García et al., 2018) indican que entre las propuestas más acertadas a esta delimitación es la propuesta por Karasek (1979) quien propuso que las principales fuentes de estrés laboral, surgen en su primera propuesta de dos características básicas: Demandas laborales, que son las exigencias psicológicas que tiene el trabajo sobre el individuo, y el control que se tiene sobre ellas, hace referencia a cómo el individuo afronta dichas responsabilidades, éstas a su vez tiene dos componentes: La autonomía y el desarrollo de habilidades.

La propuesta de este modelo, es relacionar el estrés con la tensión de los factores psicológicos (mental strain), con ello se desea explicar que un trabajador va a experimentar tensión psicológica cuando las exigencias laborales asignadas sean elevadas y presente poco control profesional para manejar la situación. (Karasek et al., 1990)

Posteriormente a este modelo propuesto, se agregó una tercera dimensión: Apoyo social, el cual es un amortiguador del efecto que ejerce el estrés en la salud del individuo. (Johnson et al., 1994). Esta dimensión explica dos componentes: Apoyo emocional y soporte instrumental, influyen en el clima social donde labora el profesional relacionándolo tanto con los compañeros como sus superiores. (FernándezArata et al., 2017; Carrillo-García et al., 2018)

Para Tezcan (2019), la satisfacción laboral es un aspecto imprescindible para que los individuos puedan desempeñarse en las organizaciones, el estrés laboral está en la cima de estos factores, un cierto nivel de estrés es considerado normal, pero cuando el trabajo es cada vez más estresante y recargado de acciones puede causar problemas crónicos en los trabajadores.

En este sentido, Chiavenato (2009) indica que las organizaciones deben alcanzar elevados niveles de desempeño, esta se logra mediante la mejora continua de los talentos humanos y a su vez de la respuesta a las necesidades de los individuos, de su autocontrol, por tanto, la motivación y el desempeño están íntimamente ligados para el logro de la satisfacción y el buen desenvolvimiento de los individuos en su centro de labores.

Para García (Citado en Chávez, 2014), considera que el desempeño son las acciones observables y medibles que realizan los individuos los cuales son importantes para el logro de objetivos de toda organización.

Según Martínez-Chairez et al., (2016), la evaluación del desempeño docente dentro del aula debe ser en un panorama real, de lo que se hace y no se hace, con la firme intencionalidad de lograr una 
educación de calidad, con el resultado se debe tomar decisiones aplicadas a la práctica profesional para las mejoras necesarias.

También definiremos términos importantes que involucran el buen desempeño de toda persona; así tenemos competencia, definida como las habilidades y facultades del ser humano de combinar sus capacidades para lograr un propósito específico, pertinente y ético. (Ministerio de educación, 2016)

Para Dimaté et al., (2017) la evaluación del desempeño docente debe reflejar la interacción de las vivencias y evidencias de los docentes, por ello debe existir una coherencia entre la claridad conceptual de la medición y el propósito que se quiere alcanzar, para delimitar los agentes que intervienen en dicho proceso, esto permitirá diseñar instrumentos adecuados que integren desde la política nacional educativa hasta los objetivos contextualizados; aplicando los criterios de calidad para lograr un país con una mejor calidad educativa de nuestros estudiantes.

En la investigación realizada por Achata et al., (2018), indican que la Dirección nacional de Evaluación docente dispuso una evaluación para 25 mil docentes de nivel inicial para el 2017, para el nivel primario supera los 110 mil docentes en el 2018, esta evaluación comprendió doce aspectos, seis de ellos fueron evaluados por observación de sesiones de aprendizaje y a estudiantes a cargo del docente, incluyendo las fichas de evaluación (MINEDU RSG Nº 078, 2017; MINEDU, 2017b) y, otras seis rúbricas que valorarán el accionar del docente en el proceso pedagógico, el cual debe promover el aprendizaje, razonamiento, creatividad y el desarrollo del pensamiento crítico.

Ahora bien, según el Ministerio de Educación (2012) dentro del Marco del buen Desempeño Docente, planteó un programa que busca contribuir en mejorar el desempeño docente de EBR en todos los niveles. Este programa tiene como propósito impulsar y promover que el docente sea revalorado, fortaleciendo su imagen profesional y estimulando el perfeccionamiento de su práctica pedagógica.

Por tanto, esta investigación considera importante estudiar el estrés en los docentes de las Instituciones Educativas de Chepén y su asociación con el desempeño, tomando en cuenta los factores que hacen que la práctica educativa se vuelva excesiva, afectando directamente la salud física, integral y emocional, repercutiendo en el desempeño de sus labores y la salud de dicho individuo; de esta manera aportar en la mejora educativa. Y se apoya en la investigación de Chiang et al (2013), cuyo objetivo fue hacer un análisis de los efectos que pueden ocasionar el estrés laboral y su influencia en el desempeño docente, apoyándose en las teorías de Karasek y Johnson, las cuáles son también asumidas en el presente trabajo.

El objetivo general planteado fue determinar si existe relación entre el estrés laboral y el desempeño de las Instituciones Educativas de Chepén - 2018; y los objetivos específicos; identificar el nivel de estrés laboral en los docentes de las Instituciones Educativas de Chepén; identificar el desempeño 
laboral en los docentes de las Instituciones Educativas de Chepén; identificar la asociación entre la dimensión apoyo en el trabajo, demandas psicológicas y control sobre el trabajo con el desempeño laboral de las Instituciones Educativas de Chepén.

\section{MÉTODO}

\subsection{DISEÑO DE INVESTIGACIÓN}

Descriptivo Correlacional simple prospectivo de corte transversal ya que el estudio determinó el nivel de asociación del estrés laboral y el desempeño de los docentes. (Hernández et al., 2010)

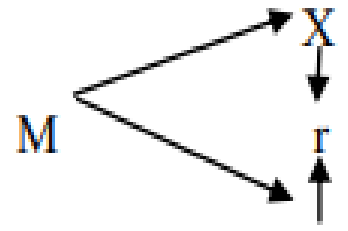

Y
M: Muestra,

$\mathrm{X}: \quad$ Estrés laboral

Y: Desempeño docente

r: Relación de asociación

\subsection{POBLACIÓN, MUESTRA Y MUESTREO}

La población lo constituyeron todos los docentes del área urbana y rural de las instituciones públicas de la provincia de Chepén año 2018, es decir aquellos docentes que ejercían su profesión durante el periodo de la investigación.

La muestra, los docentes de 3 instituciones educativas públicas, siendo del área urbana 55 docentes del I.E. N N 80830 “Zoila Hora de Robles”, 30 docentes del I.E. Carlos Olivares y; del área rural 40 docentes de la I.E “Divino Maestro” de la provincia de Chepén, haciendo un total de 125 docentes.

El muestreo fue no probabilístico por conveniencia (Malhotra, 2008), y se trabajó con todos los docentes a los que se pudo acceder y estaban disponibles el día de la toma de información.

\subsection{TÉCNICAS E INSTRUMENTOS DE RECOLECCIÓN DE DATOS}

Se utilizó como técnica la encuesta y como instrumento dos cuestionarios, el primero con 18 ítems y se calificó como Estrés bajo de 0 a 30 puntos, medio de 31 a 60 puntos y alto de 61 a 90 puntos.

El segundo cuestionario para el desempeño laboral, se determinó mediante la ficha de evaluación de desempeño por el método de escala gráfica que consta de 21 ítems, el cual se calificó como malo de 0 a 35 puntos, regular de 36 a 70 puntos y bueno de 75 a 105 puntos.

Estos instrumentos tanto para estrés laboral como para desempeño están debidamente validados por juicio de expertos y arrojaron un alfa de Cronbach de 0.787 y 0.912 respectivamente lo que significa un nivel respetable y muy alto en su confiabilidad. 


\subsection{MÉTODOS DE ANÁLISIS DE DATOS:}

Los datos se analizaron mediante el programa SPSS versión 25, y el programa Microsoft Excel, que permitió elaborar la base de datos, tablas estadísticas y el análisis de la contrastación de hipótesis para determinar la asociación entre las variables analizadas.

\section{RESULTADOS}

Tabla 1

Correlación entre el estrés y el desempeño laboral de los docentes de las Instituciones Educativas de Chepén en el año 2018

\begin{tabular}{lcc} 
& Rho de Spearman & Desempeño Laboral \\
\hline Estrés Laboral & Rho &,$- 212^{*}$ \\
\cline { 2 - 3 } & Sig.(p) & .019 \\
\cline { 2 - 3 } & $\mathrm{n}$ & 125 \\
\hline
\end{tabular}

* La correlación es significativa en el nivel 0,05

Fuente: Cuestionario aplicado a los docentes.

En la tabla 1 se observa que el valor de $\mathrm{P}$ es menor a 5\% por lo cual indica que existe relación entre el estrés y desempeño laboral, así mismo el coeficiente de correlación arrojó un valor de -0.212 , indicando que el tipo de correlación es inverso, esto quiere decir que los docentes de las instituciones educativas evidencian un menor desempeño laboral cuando son sometidos a trabajo bajo presión para el cumplimiento de sus objetivos asignados por la institución donde laboran.

Tabla 2

Nivel de Desempeño Laboral en docentes de las Instituciones Educativas de Chepén en el año 2018

\begin{tabular}{ccc}
\hline NIVEL & DOCENTES & $\%$ \\
\hline MALO & 0 & 0 \\
REGULAR & 52 & 42 \\
BUENO & 73 & 58 \\
\hline TOTAL & 125 & 100 \\
\hline
\end{tabular}

Fuente: Cuestionario aplicado a los docentes

En la tabla 2 se observa que el 58\% de los docentes presentan un buen desempeño laboral, esto garantiza que las instituciones educativas brindan una enseñanza de calidad a sus estudiantes, contando con profesionales capaces de desempeñar una buena labor educativa. 
Tabla 3

Nivel de Estrés Laboral en docentes de las Instituciones Educativas de Chepén en el año 2018

\begin{tabular}{ccc}
\hline NIVEL & DOCENTES & $\%$ \\
\hline BAJO & 0 & 0 \\
MEDIO & 77 & 62 \\
ALTO & 48 & 38 \\
\hline TOTAL & 125 & 100 \\
\hline & Fuente: Cuestionario aplicado a los docentes
\end{tabular}

En la tabla 3 se evidencia que el $62 \%$ de los docentes presentan un cuadro de estrés medio, eso indica que la carga laboral está generando presencia de estrés lo cual si esto va en aumento, podría afectar directamente a su desempeño como docentes.

Tabla 4

Correlación entre las dimensiones Apoyo en el trabajo, Demanda psicológica y Control sobre el trabajo con el desempeño laboral.

\begin{tabular}{|c|c|c|}
\hline Rho de Spearman & & DESEMPEÑO LABORAL \\
\hline \multirow{3}{*}{ APOYO EN EL TRABAJO } & Rho & $-0,21 *$ \\
\hline & Sig. & 0.02 \\
\hline & $\mathrm{N}$ & 125 \\
\hline \multirow{3}{*}{ DEMANDA PSICOLÓGICA } & Rho & 0.15 \\
\hline & Sig. & 0.09 \\
\hline & $\mathrm{N}$ & 125 \\
\hline \multirow{3}{*}{ CONTROL SOBRE EL TRABAJO } & Rho & 0.09 \\
\hline & Sig. & 0.31 \\
\hline & $\mathrm{N}$ & 125 \\
\hline
\end{tabular}

Fuente: Cuestionario aplicado a los docentes

Finalmente, en la tabla 4 se evidencia que solo las dimensiones Apoyo en el trabajo y Demanda psicológica han arrojado un $\mathrm{P}$ valor menor al 0.05 , por lo cual se demuestra que tienen relación con el desempeño laboral de los docentes en las instituciones educativas de Chepén.

\section{DISCUSIÓN}

La investigación se orienta a analizar la relación que existe entre el estrés laboral y el desempeño de los docentes de las instituciones educativas de Chepén, el cual tuvo como resultado que existe una relación inversa y significativa entre ambas variables. La tabla 1 concuerda con Zuniga et al., (2018) quienes analizaron el nivel de estrés a docentes de un colegio público regional de Chile, encontrando que dichos docentes tenían un nivel medio de estrés, así nuestra investigación nos indica que a pesar de que la relación es débil, es significativa, a esto acotamos que la exigencia en los trabajos y otros factores como el miedo a perder el puesto laboral, también influyen sobre el desempeño docente, por lo que el trabajador pese a estar estresado se esfuerza por cumplir con el desarrollo de sus sesiones y labor docente. 
En la tabla 2, podemos apreciar que la mayoría (58\%) de docentes se encuentran en un nivel alto de desempeño laboral, este resultado es evidenciado por la gestión de sus directores quienes están en constante coordinación con los docentes de dichas instituciones motivando a mejorar y exigiendo para cumplir con el desempeño. Martínez-Chairez et al., (2016), indican que la evaluación del desempeño docente debe ser en un panorama real, evaluando lo que realmente se hace, para poder tomar decisiones que ayuden a mejorar la calidad educativa. Por ello el Ministerio de Educación (2012), implementó un programa que busca contribuir el buen desempeño docente en Educación Básica Regular en todos los niveles con el propósito de que el docente sea revalorado, tenga una imagen digna y que busque su desarrollo y perfección en el desarrollo de su labor en la institución educativa y calidad educativa.

Asimismo, la tabla 3 muestra que los docentes de las instituciones educativas de Chepén evidencian un nivel de estrés medio (62\%) en su mayoría ocasionado por la misma exigencia por parte de los directores, supervisores o jefes de áreas quienes en algunas ocasiones no están debidamente preparados y lejos de ejercer el apoyo que necesitan los docentes, sólo emiten críticas o plantean situaciones generales y no tienen en cuenta la contextualización o realidad de los agentes de la institución educativa, también influye los cambios constantes del sistema educativo, los cuales son copias de otros sistemas internacionales y no reflejan la realidad de nuestras instituciones, las horas de trabajo en casa para diseñar las sesiones de clases y presión en el cumplimiento de las políticas educativas actuales, lo que concuerda con Leal (2006), quien indica tres tipos de estrés; agudo, episódico y crónico, cada uno de ellos diferenciados por las circunstancias del estado de estrés en el que se encuentre. Asimismo, Ontiveros et al., (2019), quien define el término estrés como una respuesta del individuo ante cualquier estímulo o situación estresante.

Y por último la tabla 4, se aprecia una correlación inversa, de grado débil pero significativa $(p<0.05)$ solo entre la dimensión apoyo en el trabajo y el desempeño laboral de los docentes de las Instituciones Educativas de Chepén haciendo referencia al modelo propuesto por Karasek(1979), citado por los investigadores (Chiang et al., 2013; Fernández-Arata et al., 2017; Carrillo-García et al., 2018 en la que se identifica el estrés como la tensión psicológica que el profesional experimenta cuando las demandas laborales son altas y tiene escaso control para ejercer sobre la situación, a esto se suma el apoyo social propuesta por Johnson et al., (1994), el cual es un amortiguador del efecto del estrés en la salud, esta se refiere al apoyo emocional y soporte instrumental que influye en el clima social donde labora el profesional relacionándolo tanto con los compañeros como sus superiores.

Es importante mencionar que la UGEL Chepén realiza capacitaciones como apoyo a la labor docente las cuales son difundidas por los directores de las instituciones educativas. 


\section{CONCLUSIONES}

Existe asociación inversa entre el estrés laboral y el desempeño laboral de los docentes de las Instituciones Educativas de Chepén en el año 2018.

Se determinó que la mayoría de docentes presentan un nivel de estrés laboral medio

Se determinó que la mayoría de docentes presentan un nivel de desempeño bueno.

De las dimensiones analizadas del estrés, solo existe relación y de tipo inversa entre la dimensión apoyo en el trabajo y el desempeño laboral de los docentes de las Instituciones Educativas de Chepén en el año 2018. 


\section{REFERENCIAS}

Achata, C. y Quispe, Y.A. (2018). El Currículo Nacional y el desempeño docente. Revista de Investigaciones de la Escuela de Posgrado, 7(2), 598-606. doi: 10.26788/riepg.2018.2.84

Acosta, A. C., Jimenez, L. K., Redondo, M. y Pulido, E.G. (enero-junio, 2019). Estrés ocupacional y evaluación de desempeño en docentes universitarios del departamento del Cesa, Colombia. Revista Encuentros, 17(01), 24-33. http://ojs.uac.edu.co/index.php/encuentros/article/view/1595/1221

Carrillo-García, C., Ríos-Rísquez, M., Escudero-Fernández, L. y Martínez-Roche, M. (2018). Factores de estrés laboral en el personal de enfermería hospitalario del equipo volante según el modelo de demanda$\begin{array}{llll}\text { control-apoyo. } & \text { Enfermería } & \text { global. } & \text { 304-324. }\end{array}$ https://revistas.um.es/eglobal/article/view/277251/226341

Chávez, A., (julio, 2014), Gestión del desempeño en las organizaciones educativas. Horizonte de la ciencia, $4(6)$, $75-81$. https://www.researchgate.net/publication/318842957_Gestion_del_desempeno_en_las_organizaciones_ educativas

Chiang, M., Gómez, N. y Sigoña, M. (2013). Factores psicosociales, stress y su relación con el desempeño: comparación entre centros de salud. Salud de los trabajadores. 21(2). 11-128. https://www.redalyc.org/pdf/3758/375839307002.pdf

Chiavenato, I (2009). Administración de recursos humanos.

Dimaté, C., Tapiero, O., González, C. I., Rodriguez, R., y Arcila, M. A. (julio-diciembre, 2017). La evaluación del rendimiento docente. Revista Folios. (46), 83-95. https://www.redalyc.org/articulo.oa?id=3459/345951474007

Fernández-Arata, J. M. y Calderón-De la Cruz, G. (2017). Modelo Demandas-Control-Apoyo social en el estudio del estrés laboral en el Perú. Revista Médica Herediana, 28(4), 281-282. https://www.redalyc.org/articulo.oa?id=3380/338053958014

Gallardo-Lópes, López-Noguero y Gallardo-Vásquez (mayo - agosto, 2019). Análisis del síndrome de burnout en docentes de educación infantil, primaria y secundaria para su prevención y tratamiento. Revista Electrónica Educare. 23(2), 1-20. https://www.scielo.sa.cr/scielo.php?pid=S1409$42582019000200324 \&$ script=sci_abstract\&tlng=es

Hernandez, Fernandez, y Baptista. (2010). Metodologia de la Investigación Cientifica. Mexico: Mc Graw Hill/ Interamericana editores S.A. de C.V

Johnson, J.V. y Hall, B.M. (1994). Social support in the work environment and cardiovascular disease. Social Support and Cardiovascular Disease. 145-166. https://link.springer.com/chapter/10.1007/978-14899-2572-5_7

Karasek, R. A. (1979). Job Demands, Job Decision Latitude, and Mental Strain: Implications for Job Redesign. Administrative Science Quarterly, 24(2), 285-308. https://www.jstor.org/stable/2392498?seq=1

Karasek R. A, Theorell T. (1990). Healthy Work, Stress, Productivity and the Reconstruction of Working 
Life. Basic Books: New York

Ley de Reforma Magisterial (2013). Ley $N^{\circ}$ 29944. Reglamento de la Ley de la reforma magisterial D.S. $N .^{\circ}$ 004-2013-ED. http://www.minedu.gob.pe/reforma-magisterial/pdf-ley-reforma-magisterial/leyreforma-magisterial-29944.pdf

Leal, I. (2006), El estrés: Cómo nos afecta. Revista Científica General José María Córdova, 4(4), 56-58. https://www.redalyc.org/pdf/4762/476259067015.pdf

Lemos, M., Calle, G., Roldán, T., Valencia, M., Orejuela, J. J. y Román-Calderón, J. P. (enero-junio, 2019). Factores psicosociales asociados al estrés en profesores universitarios colombianos. Diversitas: Perspectivas en Psicología, 15(1), 61-72. http://www.scielo.org.co/scielo.php?pid=S1794 $99982019000100061 \&$ script=sci_abstract\&tlng=es

Luceño L., Martín, J., Jaén, M. y Díaz, E. M. (2005). Evaluación de factores psicosociales en el entorno laboral. EduPsykhé Revista de Psicología y $\begin{array}{lllll} & \text { Psicopedagogía, } & 4(1), & 19 & -42\end{array}$ https://journals.ucjc.edu/EDU/article/view/3761/2707

Malhotra, N. K. (2008). Investigación de Mercados. Naucalpan de Juárez, México: Pearson Prentice Hall.

Martínez-Chairez, G., Guevara-Araiza, A. y Valles-Ornelas, M. (julio-diciembre, 2016). El desempeño docente y la calidad educativa. Revista $R a \quad X i m h a i, \quad 12(6), \quad 123-134$. http://redib.org/Record/oai_articulo1489241-el-desempe\%C3\%B1o-docente-y-la-calidad-educativa

Ministerio de educación. (2012). Marco del buen Desempeño Docente. Para mejorar tu práctica como maestro y guiar el aprendizaje de tus estudiantes. http://www.minedu.gob.pe/pdf/ed/marco-de-buendesempeno-docente.pdf

Ministerio de Educación. (2016). Currículo Nacional de la Educación Básica. http://www.minedu.gob.pe/curriculo/pdf/curriculo-nacional-2016-2.pdf

MINEDU. (2017a). Resolución de Secretaría General. Recuperado de https://cdn.www.gob.pe/uploads/document/file/111006/_078-2017-MINEDU_-_29-032017_12_26_49_-RSG_N_078-2017-MINEDU.pdf

MINEDU. (2017b). Resolución de Secretaría General $N^{\circ}$ 141-2017-MINEDU Norma que regula la Evaluación Ordinaria de Desempeño para profesores de Instituciones educativas de nivel inicial de la Educación Básica Regular de la Carrera Pública Magisterial. http://evaluaciondocente.perueduca.pe/media/11496168364RSG141-2017-MINEDU-Aprueba-NTEvaluacion-desempe\%C3\%B1o-ordinaria-EBR-incial.pdf

Muñoz, E. J., Mantilla, M.I., Gonzalez, M., Cruz, J. W. y Rodriguez, D. (2020). Validación aplicativo móvil para detección del Burnout en Docentes Bogotá. South Florida Journal of Development. 1(4). 320331. https://southfloridapublishing.com/ojs/index.php/jdev/article/view/70/83

Noblet, A. y Lamontagne A.D. (2006). The role of workplace health promotion in addressing job stress. Health Promotion International, 346-353. https://pdfs.semanticscholar.org/c6ce/dee2835c3152c105a1f6955cbf6ee45b6318.pdf?_ga=2.199620715. 477683189.1613874642-365329789.1613397977 
Ontiveros, L.G. y Hernández, S. M. (enero-junio, 2019), El estrés laboral en trabajadores a nivel primaria. Estudios clínicos e Investigación Psicológica, 9(17), 93-101. https://issuu.com/revistacecip/docs/vol_9_no_17_enero-junio_2019

Organización Internacional del Trabajo. (2016). Estrés en el trabajo: Un reto colectivo. Día Mundial de la seguridad y la salud en el trabajo 26 de abril 2016. https://www.ilo.org/wcmsp5/groups/public/--ed_protect/---protrav/---safework/documents/publication/wcms_466549.pdf

Rodríguez, J. A., Guevara, A. y Viramontes, E. (abril-setiembre, 2017). Síndrome de burnout en docentes. IE Revista de investigación educativa de la REDIECH, 8(14), 41-67. http://www.scielo.org.mx/pdf/ierediech/v8n14/2448-8550-ierediech-8-14-45.pdf

Stavroula, L., Griffits, A. y Cox, T. (2004). Estrategias sistemáticas de solución de problemas para empleadores, personal directivo y representantes sindicales. La Organización del trabajo y el estrés. 3, 1-27. https://www.who.int/occupational_health/publications/en/pwh3sp.pdf

Tezcan, H. (2019). The Mediation Role of Toxic Leadership in the Effect of Job Stress on Job Satisfaction. International Journal of Business. https://www.researchgate.net/profile/Htezcan_Uysal/publication/331249551_The_Mediation_Role_of_ Toxic_Leadership_in_the_Effect_of_Job_Stress_on_Job_Satisfaction/links/5ce475b5a6fdccc9ddc4a97f /The-Mediation-Rzole-of-Toxic-Leadership-in-the-Effect-of-Job-Stress-on-Job-Satisfaction.pdf

Zuniga-Jara, S. y Pizarro-Leon, V. (2018). Mediciones de estrés laboral en docentes de un colegio público regional chileno. Información tecnológica. $29(1) . \quad 171-180$. https://scielo.conicyt.cl/scielo.php?script=sci_arttext\&pid=S0718-07642018000100171 